\title{
Atomic approach to general relativity
}

Discussions linking quantum theory and gravity can be traced at least back to Einstein, who famously argued that, if energy is conserved, a photon must be red-shifted on climbing through a gravitational field. This argument alone shows that special relativity cannot adequately describe a universe with gravity and, in the 1960s, experiments using the Mossbauer effect, another quantum phenomenon, confirmed it. Many other manifestations of general relativity have now been observed, the most demanding typically involving planets or stars, or light moving over astronomical distances.

However, our understanding of the relationship between gravity and quantum physics remains speculative. So it is surprising that quantum effects may be put to use again in improving tests of general relativity - they may even be able to detect general relativistic influences in atomic motion within human-scale laboratories.

For atoms falling near the Earth's surface, the magnitude of the relativistic component of the gravitational field is only about
$10^{-15} \mathrm{~g}$, which would seem to make detection almost hopeless. But the accuracy of atom interferometry may be up to the challenge. A recent proposal suggests that this technique should be able to probe general relativity at the $10^{-15} \mathrm{~g}$ level and beyond (S. Dimopoulos et al. http://arxiv.org/abs/gr-qc/0610047). Devices tracking the coherent dynamics of atomic beams may even surpass large-scale astrophysical tests in accuracy.

The basic idea is to fire coherent pulses of cold atoms upwards and then let them fall. Laser pulses interacting with the atoms can recreate the logic of a Mach-Zender interferometer. A series of pulses can act first to split the beam, sending its components along distinct space-time paths, and then to recombine the components for the detection of interference fringes. A rough estimate of the numbers shows that with such a device — roughly $10 \mathrm{~m}$ in height, with atoms falling for a little more than $1 \mathrm{~s}$ and the results from repeated pulses integrated over the course of a day - general relativistic effects could be just within reach.

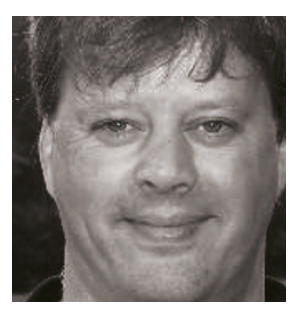

This proposed experiment seems also to offer a natural means for distinguishing fundamentally different effects. Departures from newtonian gravity arise in part from the nonlinearity of general relativity, as the field is itself a source for further gravity. Moving atoms also contribute to the field in proportion

THE BASIC

IDEA IS TO FIRE COHERENT

PULSES OF

COLD ATOMS

UPWARDS

AND THEN LET

THEM FALL. to their kinetic energy, which also gravitates. As Dimopoulos and colleagues argue, however, these distinct contributions should scale differently with the physical parameters of an experiment - the inital atomic velocity, the time of fall, and so on - suggesting that systematic experiments could probe them separately.

All of which is encouraging, given recent findings concerning dark matter and the apparently accelerating expansion of the Universe, which hint that we may still have a lot to learn about general relativity. We may for a long time lack any way to unify quantum theory with gravity, but understanding the one may give us unprecedented means for learning about the other.

Mark Buchanan

\section{Another year over...}

It's been a tumultuous year for science and society, and, in my final column for Nature Physics, I'll briefly review the latest news on some of the issues I have written about over the past year.

First, on missile defence: bad news. The US decision to forego direct non-proliferation talks with North Korea clearly contributed to their decision to explode a nuclear device in October, leading to misguided calls to deploy even more non-functional missile interceptors, at great expense and with no gain in security. One can only hope that rationality will prevail and that countries will not feel driven to acquire nuclear weapons as their best defence against possible pre-emptive actions by the US or its allies.

For the Hubble Space Telescope, it's good news: NASA has just announced a shuttle mission in 2008 to repair and upgrade the telescope. There was no reason to forego this single mission to one of the most important bits of scientific machinery in space, when more than 20 missions, of almost equal hazard, were planned to maintain the largely useless International Space Station.

More bad news, however, on scientific integrity. It seems that, in the US, political interference in the results of scientific investigations continues. The Assistant Secretary for Fish and Wildlife, a political appointee with no biological training, is reported to have rewritten numerous scientific reports, changing their conclusions. At the same time, the Bush administration's censorship of global warming research is

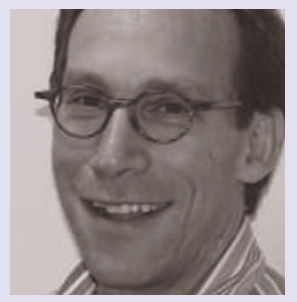

IT'S BEEN A TUMULTUOUS YEAR FOR SCIENCE AND SOCIETY. under investigation. Let's hope that science might soon be the basis of sound public policy once again.

For fundamental physics, I'm predicting good news. Starting next year, the Large Hadron Collider will no doubt produce surprises, and my hope is that they point to something other than the currently fashionable models for new physics. The next frontier of astrophysical and cosmological investigation - gravitational waves - should become empirical science by 2010 or so, with improvements to LIGO underway. But I expect that one of the biggest mysteries, the nature of dark energy, will remain so for the foreseeable future. But then, it's the unexpected, and hence unpredictable, that makes physics so exciting.

Lawrence M. Krauss 